\title{
Quantifying the durability of a friction-reducing surface with recoverable super-hydrophobicity
}

\author{
Liliane C.C. Auwerter, Christopher Cheeseman, \\ Michael R. Templeton and Maarten van Reeuwijk ${ }^{1}$
}

\begin{abstract}
The durability of super-hydrophobic surfaces in fully immersed conditions is a major obstacle to their application in engineering applications. We perform an experimental study to measure the friction factor $f_{d}$ as a function of time for a new super-hydrophobic surface that is capable of recovering the Cassie-Baxter wetting state. Values of $f_{d}$ were obtained by measuring the pressure drop and volume flux of a turbulent water flow in a 1.5 metre long duct containing one super-hydrophobic wall. The Reynolds number of the flow was approximately $4.5 \times 10^{4}$ for all experiments. Reductions in $f_{d}$ were $29-36 \%$ relative to a hydraulically smooth surface. The Cassie-Baxter state could be recovered blowing air through the porous surface for 10 minutes. The durability of the drag-reduction, as quantified by the relaxation time $T$ in which the surface loses its superhydrophobic characteristics, were measured to be between 10-60 minutes depending on the initial head. The relaxation time $T$ was highly dependent on the pressure difference across the surface. In contrast to models based on Darcy flow through a porous medium, the study indicates that there seems to be a critical pressure difference beyond which the Cassie-Baxter state cannot be sustained for the material under consideration.
\end{abstract}

\section{INTRODUCTION}

The transportation of dense fluids is an energy-intensive activity. To deliver water in pipelines from the treatment plant via a pressurised distribution system to the end-user the water industry consumes globally 180 TWh of energy (IEA, 2016). Major energy losses $(\Delta H)$ occur in water distribution as a result of friction with pipe walls. The power $P_{w}$ needed to maintain a volume flux $Q\left(\mathrm{~m}^{3} / \mathrm{s}\right)$ of fluid with density $\rho\left(\mathrm{kg} / \mathrm{m}^{3}\right)$ for a pipe with Darcy-Weissbach friction factor $f_{d}$ is given by (Nakayama, 2018):

$$
P_{w}=\alpha f_{d} U^{3}
$$

where $\alpha=A L / 2 D$ encapsulates the pipe geometry, $U$ is the fluid velocity, $A$ is the crosssectional area, $L$ is the pipe length and $D$ is the pipe diameter. The strongest energy reduction can be obtained by reducing the velocity in the pipe, for example by making the pipe diameter $D$ very large. However, apart from the costs associated with very large diameter pipes, low velocities will cause sedimentation which is undesirable (Bennett and Glaser, 2011).

Assuming that the velocity $U$ and diameter $D$ are determined by other considerations, the main parameter to reduce energy consumption is to reduce the pipe friction factor $f_{d}$. The friction factor is a function of the flow Reynolds number $R e=U D / \nu$ and the ratio $\epsilon / D$,

\footnotetext{
${ }^{1}$ Department of Civil and Environmental Engineering, Imperial College London, South Kensington, London, United Kingdom SW7 2AZ
} 
where $\nu$ is the kinematic viscosity and $\epsilon$ is the equivalent sand grain roughness (Moody, 1944). The best one can do with a conventional material is to employ a surface which is hydraulically smooth.

A promising methodology to reduce drag further than that of a hydraulically smooth surface is by using superhydrophobic surfaces. Superhydrophobicity is found in nature in Lotus leaves (Nelumbo nucifera), which present self-cleaning properties due to their hierarchical surface structure covered in a wax of low surface energy (Nosonovsky and Bhushan, 2008). This results in dirt particles on the surface being picked up by droplets of water due to the low adhesion of dirt particles and water on the highly water repellent surface. Super-hydrophobic materials have water contact angles greater than $150^{\circ}$ and are associated with surfaces that present a surface structure that can be engineered or roughened randomly (e.g. by deposition of nano-particles), and combined with low surface free energy, exist in the Cassie-Baxter wetting state (Nosonovsky and Bhushan, 2008). This paper focuses on super-hydrophobic surfaces with randomly arranged surface elements that create a roughened solid surface. In the Cassie-Baxter state a liquid resting on the roughened solid surface is partially suspended by the air pockets trapped on the solid surface between the roughness elements. This state is to be contrasted with the Wenzel state, in which there are no air pockets between the solid-liquid interface, and the liquid is fully in contact with the solid roughness elements.

Super-hydrophobic surfaces in the Cassie-Baxter state reduce the surface-liquid contact area, and thus decrease friction. Indeed, as air is approximately 50 times less viscous than water, the shear stress on parts of the surface where air is present will also be smaller by that factor and can thus be considered shear-free (Ybert et al., 2007; Davis and Lauga, 2009). The use of super-hydrophobic surfaces in turbulent flows has been investigated numerically and experimentally, showing drag reduction and apparent slip on the surface due to the shearfree areas where the air is present (Costantini et al., 2018; Daniello et al., 2009; Min and Kim, 2004; Yao et al., 2015). The apparent slip is quantified by means of a slip length $\ell_{s}$ by matching measurements to an effective boundary condition $u_{w}=\ell_{s} d u /\left.d z\right|_{w}$ (Navier, 1823), where $u_{w}$ is the slip velocity and $d u /\left.d z\right|_{w}$ is the velocity gradient at the wall. The limits of $\ell_{s} \rightarrow \infty$ and $\ell_{s} \rightarrow 0$ represent free-slip and no-slip boundary conditions respectively. Typical values of $\ell_{s}$ for superhydrophobic surfaces are in the nano- to micrometer scale, depending on the viscosity, velocity and direction of the fluid, surface dimension and surface pattern, among others. For turbulent flows, slip lengths of 70 to $120 \mu \mathrm{m}$ have been found experimentally at $\operatorname{Re} \geq 4,000$ (Daniello et al., 2009). A slip velocity of $1.4 \mathrm{~cm} / \mathrm{s}$ for $\operatorname{Re}$ $=11,000$ was observed for water flowing on a super-hydrophobic hydrofoil (Gogte et al., 2005). Studies presenting apparent slip on super-hydrophobic walls have employed superhydrophobic coatings (Guan et al., 2015; Du et al., 2017), and super-hydrophobic coated surfaces with micro-sized features (Gogte et al., 2005; Daniello et al., 2009).

Experimental studies measured drag reduction in pipes and channels with super-hydrophobic walls by measuring pressure drop and velocity of the fluid. For instance, Daniello et al. (2009) obtained up to $50 \%$ drag reduction in a experiment of water flowing under turbulent conditions over engineered super-hydrophobic surfaces. Here drag reduction $(D R \%)$ is defined as $D R \%=1-\left(f_{d} / f_{0}\right) \times 100$, where $f_{0}$ is the friction factor to which $f_{d}$ is being compared. In Daniello et al. (2009), the pressure drop was obtained using the height difference of water in piezometric tubes, and the velocity of water close to the wall was obtained using particle 
image velocimetry. Yao et al. (2015) obtained $12 \%$ drag reduction in a water tunnel experiment, which was inferred using pressure drop measurement from pressure sensors. Walker et al. (2016) measured the time, and consequently velocity, of 10-30 $L$ water flowing in a super-hydrophobic pipe of $0.015 \mathrm{~m}$ diameter, reporting a friction factor reduction of $33 \%$.

Given their friction-reducing nature, super-hydrophobic surfaces have great potential in the hydraulic engineering field, specially in the transport of water in pipes and in the marine industry (Fukuda et al., 2000; Yao et al., 2015; Moaven et al., 2013). The Reynolds number in these applications are in the turbulent range $(>4,000)$ and studies of water flow on superhydrophobic surfaces obtained drag reduction in experiments with $R e$ in the order of $10^{3}$ (Daniello et al., 2009), $10^{4}$ (Brassard et al., 2015) and $10^{6}$ (Moaven et al., 2013; Paik et al., 2015; Yao et al., 2015) among others. In addition, the use of super-hydrophobic surfaces in offshore oil platforms reduces formation of ice and protects the platform, ship decks, antennas and locators of ships against erosion from sea water splashes and aerosols containing high concentration of salts (Boinovich et al., 2016).

However, the super-hydrophobicity and the contact angle tend to decrease with time under continuous immersion in water due to the loss of trapped air, causing the wetting state to transition from Cassie-Baxter to Wenzel. Super-hydrophobic surfaces that transition to the Wenzel state tend to have a higher drag than a smooth surface due to their high surface roughness (Du et al., 2017). This effect implies that the friction-reducing effect of superhydrophobic surfaces is temporary, which makes them unsuitable for engineering applications in which the material is continuously immersed in fluid. There is a need, therefore, to develop superhydrophobic materials that i) are durable, in that they lose their superhydrophobicity slowly; and ii) have recoverable super-hydrophobicity, in that their superhydrophobic nature can be restored without having to dry the material. The aim of this paper is to investigate the suitability of a newly developed material with recoverable super-hydrophobicity (Auwerter et al., 2019) for use in pressurised water pipes at realistic Reynolds numbers.

The mechanism by which the air trapped in roughness elements of the super-hydrophobic surface is lost is via diffusion of air into the water and by dislocation of the air pockets (Brennan et al., 2015). The latter mechanism is particularly important for surfaces subjected to turbulent flow. Entrapped gas was observed on various rough and structured surfaces that were immersed in water under static conditions (Bobji et al., 2009; Wang et al., 2014), and the periods of retention of air were compared showing a high dependence of surface structure and the mean time for the air trapped to disappear, ranging from approximately 35 minutes for surfaces with pillars and 25 minutes for surfaces with ridges (Bobji et al., 2009), to 1 hour for micro-grooved surfaces (Wang et al., 2014). Super-hydrophobic surfaces with ridges have been shown to retain air pockets for more than 8 hours under very small static water pressure of $50 \mathrm{~mm}(\sim 490 \mathrm{~Pa})$, where the main factor to maintain the Cassie-Baxter state was the minimized environmental fluctuations such as no variation in temperature (Xu et al., 2014). Moreover, dissolved air and traces of surfactant may also increase the rate of removal of the air layer in super-hydrophobic surfaces (Mohammadi et al., 2004; Shirtcliffe et al., 2009).

A combination of techniques using super-hydrophobic materials and air injection has been previously proposed to decrease drag in boats with more durable air-solid-water interfaces (Fukuda et al., 2000). A maximum reduction of $80 \%$ in frictional resistance was achieved at a speed of $4 \mathrm{~m} / \mathrm{s}$ and $55 \%$ at $8 \mathrm{~m} / \mathrm{s}$. High drag reduction by repetitive streamwise air bubble 
a)

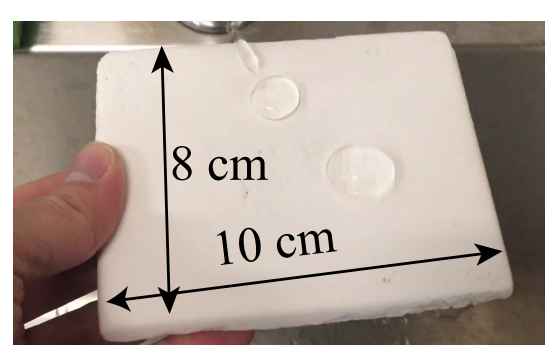

b)

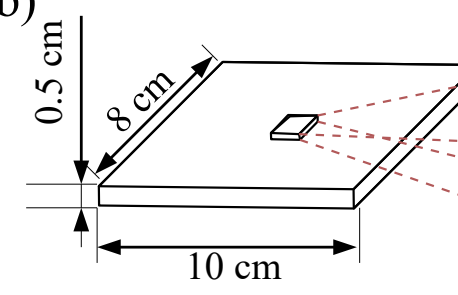

d)

c)

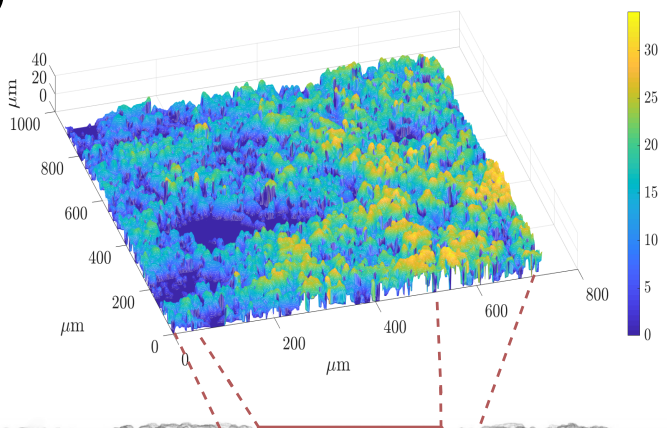

injection has been previously investigated in turbulent flow channels and ship hulls, showing reductions in drag of up to 60\% (Murai et al., 2007; Park et al., 2015; Zhang et al., 2018).

Bubble injection can be used as a means to recover the Cassie-Baxter state of a superhydrophobic surface. A similar technique of repetitive bubble injection has previously used bubble injection in an experiment that aimed to maintain the air pockets on a superhydrophobic surface under turbulent conditions, and obtained a maximum drag reduction of $20 \%$ in the Cassie-Baxter state (Du et al., 2017). Air was injected through a $0.6 \mathrm{~mm}$ hole and the air pockets were retained shortly on the surface up to only 10 seconds. By repeatedly injecting air bubbles due to the short retention of the air pockets, the CassieBaxter state near the injection hole was recovered and consequently the feasibility of use of super-hydrophobic materials improved.

An alternative to injecting bubbles in the flow is to blow air through a porous superhydrophobic surface, thereby ensuring a reasonably homogeneous replenishment of the air layer. In Auwerter et al. (2019), we reported the development of a super-hydrophobic surface with the proposed characteristics, tested under static water pressure. Figure 1 displays the porous super-hydrophobic tile used in Auwerter et al. (2019). The internal structure is completely porous and its surface presents characteristic hierarchical features similar to the micro-surface structure of the Lotus leaf. The results reported in Auwerter et al. (2019) show full recovery of the Cassie-Baxter state across the surface, indicating that the low friction properties of this surface could be maintained and/or recovered. In this study we carry out careful measurements of the friction coefficient of the porous super-hydrophobic 


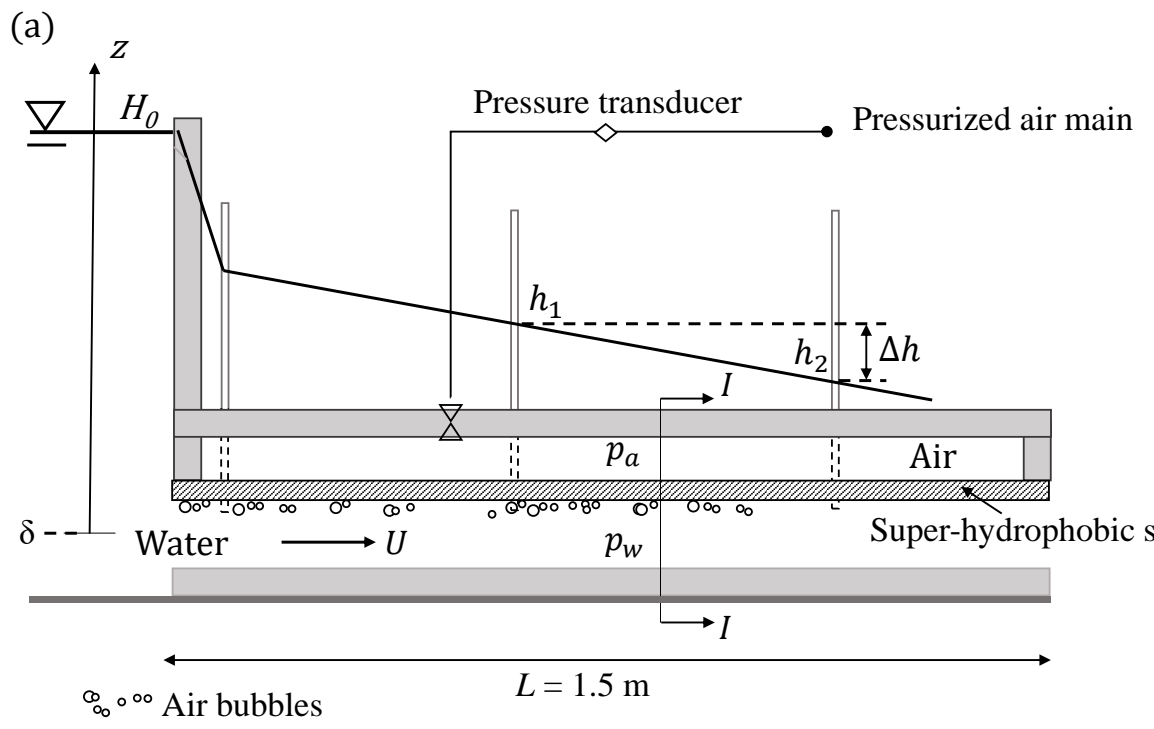

(b) $\quad I-I$

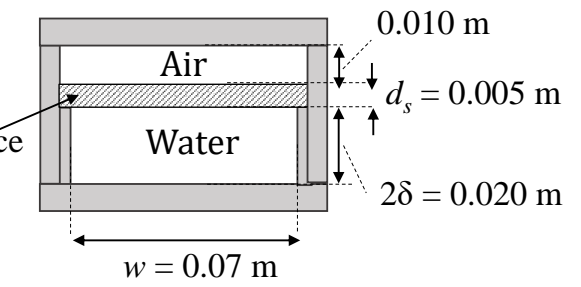

FIG. 2: Definition sketch of experimental setup, showing the duct and the superhydrophobic surface which separates the water layer from the air layer. The air layer can be pressurised to force air through the super-hydrophobic surface. (a) elevation view. (b) section I-I.

surface developed in Auwerter et al. (2019) over time under turbulent flow conditions in order to assess the recoverability of the Cassie-Baxter state and the durability of the superhydrophobic material.

The paper is organised as follows. First, the experimental methodology is described. The Results section describes the results for the evolution of the friction factor $f_{d}$ as a function of time, including an investigation of the time $T$ over which the Cassie-Baxter state is lost and how this depends on pressure $p$. Then, we explore whether the observed dependence of $T$ on $p$ can be explained from the permeability of the material. A nomenclature with all the variables and their dimensions is presented at the end of the paper.

\section{EXPERIMENTAL DETAILS}

The experimental apparatus used to determine the friction factor $f_{d}$ is shown in Fig. 2. The central element of the experimental setup was a duct of $0.055 \mathrm{~m}$ (height) $\times 0.1 \mathrm{~m}$ (width) $\times 1.5 \mathrm{~m}$ (length) made of acrylic. The duct was split into two compartments by an internal boundary which could be either super-hydrophobic or acrylic, and which separated the water below from an air layer above. The air layer could be pressurised in order to force air through the porous super-hydrophobic surface and thus recover the Cassie-Baxter state. The internal dimensions of the duct were $0.02 \mathrm{~m}$ (height) $\times 0.07 \mathrm{~m}$ (width) $\times 1.5 \mathrm{~m}$ (length)

The duct was placed in a flume of dimensions $0.3 \mathrm{~m}$ (height) $\times 0.1 \mathrm{~m}$ (width) $\times 3 \mathrm{~m}$ (length). Water was supplied at $18-19{ }^{\circ} \mathrm{C}$ from main tanks to the flume, and the height to which the water rises (initial head, $0.22 \leq H_{0} \leq 0.27 \mathrm{~m}$ ) was controlled by changing the height of the spillway hole. The initial head $H_{0}$ was adjusted at the beginning of each 


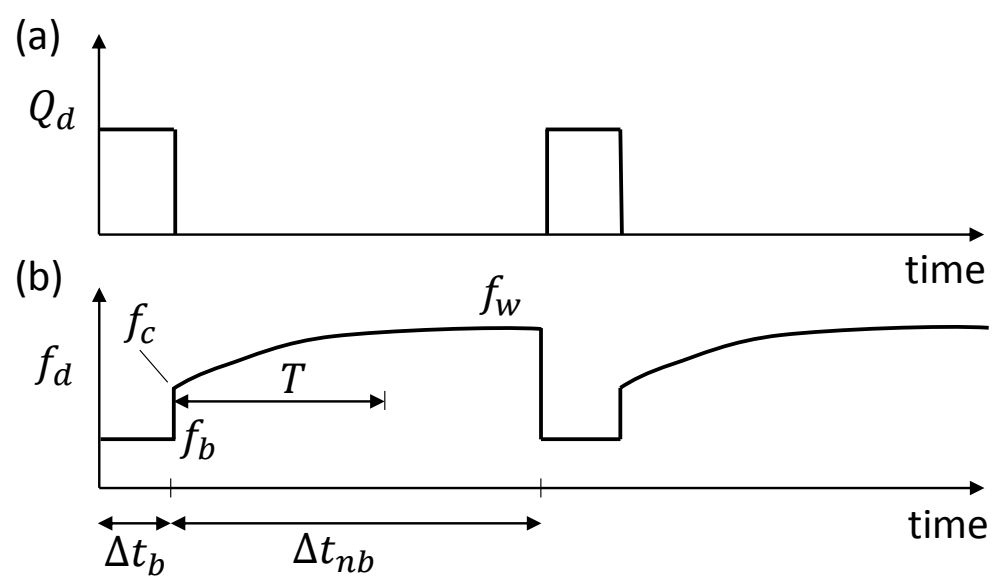

FIG. 3: Schematic of the aeration strategy (a) and it effect on the drag coefficient of the super-hydrophobic surface (b).

experiment. In the experiments with the introduction of air bubbles, pressurised air was introduced before water flowed in the duct to ensure the Cassie-Baxter state in the superhydrophobic samples, thus $H_{0}$ was adjusted whilst air was forced through the porous top layer. A flow barrier wall was placed at the upstream side of the duct and the experimental setup was sealed into the flume, as seen in Fig. 2.

Piezometric tubes were placed at $0.043,0.145,0.343,0.686,0.886$ and $1.086 \mathrm{~m}$ from the upstream side of the duct, but head measurements are presented from $0.343 \mathrm{~m}$ onwards to avoid contamination by entrance effects. The volumetric flow rate $Q$, which is crucial to measure at high accuracy, was determined by collecting the water from the duct for three minutes in a tank with surface area $A_{t}=0.87 \mathrm{~m}^{2}$. The advantage of this method is that any level of accuracy could be obtained by collecting water for a sufficiently long time. The mean velocity $U$ inside the duct was obtained simply by dividing the flow rate by the duct cross-sectional area $A$ as $U=Q / A$.

The deviation in $Q$ was used to validate the precision of measurement of $f_{d}$ of the acrylic walls. The friction factor of the acrylic was unknown, and values of $f_{d}$ of the superhydrophobic surface were expected to be similar to previous studies (Walker et al., 2016). The precision with which $f_{d}$ is measured is highly dependent on the precision measurement of $Q$, and if $f_{d}$ of acrylic were similar to $f_{d}$ of the super-hydrophobic surface, high precision measurements of $Q$ would be needed. In this experiment a maximum error $\Delta f_{d}$ of 0.005 was considered acceptable. Preliminary experiments with an acrylic top wall indicated that the relative error $\Delta Q / Q$ did not exceed $0.5 \%$, which in turn suggests that $\Delta f_{d}$ is within the accepted range of precision. A previous study has reported a similar $\Delta f_{d}$ between a super-hydrophobic and a smooth walls between flow in pipes of the same dimension at $R e$ $\geq 4,000$ (Walker et al., 2016).

A series of nine experiments were conducted. Experiments S1-3 used an acrylic top surface to verify the accuracy of the apparatus to determine the friction coefficient $f_{d}$. In addition to experiments S1-3, two other measurement sets $\mathrm{C}$ and $\mathrm{T}$, comprising of three experiments each, were performed with a super-hydrophobic top surface. In experiment set $\mathrm{C}$ continuous air bubble injection was applied through the surface for the whole duration 
of the experiment, and in experiment set $\mathrm{T}$ air bubbles were applied only prior to the start of the experiment and at specific times in order to recover the Cassie-Baxter state. The experiments were designed to measure the friction coefficient during fully aerated conditions $\left(f_{b}\right)$, immediately after the aeration was stopped and the super-hydrophobic surface was in the Cassie-Baxter state $\left(f_{c}\right)$ and the subsequent slow transition to the Wenzel state $\left(f_{w}\right)$. This process is shown diagramatically in Fig. 3. Aeration was performed for a time duration $\Delta t_{b}$, after which the super-hydrophobic surface transitions from Cassie-Baxter to Wenzel over a time-scale $T$, and the total time in which the surface was unaerated is denoted $\Delta t_{n b}$. The air hose was disconnected from the duct during $\Delta t_{n b}$ to ensure that the air pressure $p_{a}$ inside the aeration compartment was equal to the atmospheric pressure.

The aeration volume flux $Q_{d}$ was achieved by applying an air pressure $p_{a}$ to the air layer, and $Q_{d}$ was measured using a Low Pressure Drop Flow Meter (Sensirion, Zürich, Switzerland). The air pressure $p_{a}$ was obtained using a Gauge Pressure Sensor (Honeywell, New Jersey, United States) and an Arduino (Arduino, Turin, Italy) was used to record the analogue voltage output of the sensor.

Experiments C1-C3 were conducted to obtain $f_{b}$, whilst experiments T1-T3 were conducted to study the durability of the super-hydrophobicity of the surface. At $t=0 f_{c}$ was measured immediately after the experiment started. Between 0 and 1 hour $f_{d}$ was measured every 10 minutes, and between 1 and 5 hours $f_{d}$ was measured every 30 minutes. Values of $f_{w}$ were taken when $f_{d}$ was at its maximum at approximately $t=3-5$ hours. Table 1 presents the experimental conditions.

The average flow velocity $U$ in the duct depends on the applied head $H_{0}$ and the friction factor of the duct $f_{d}$. The Reynolds number was sufficient for fully turbulent flow for all experiments, but the limited range in $H_{0}$ implies that the results are constrained to $R e=$ $4.0-4.8 \times 10^{4}$, as is shown in Table 1. The Reynolds number shown in Table 1 was consistent for experiments with all smooth walls (S1-3) amongst replicates because the experiment was steady without the introduction of air bubbles or changes in the friction factor of the walls of the duct. In experiment set $\mathrm{C}$ and $\mathrm{T}$, the introduction of air bubbles affected the velocity of water flow in the duct, and in experiment set $\mathrm{T}$ the velocity of the flow changed slightly with the increase of friction factor on the interface between the super-hydrophobic material and water flow, thus affecting the Reynolds number. For comparison, a typical Reynolds number in drinking water pipes is in the order of $10^{4}-10^{6}$ for water at $20^{\circ} \mathrm{C}$ flowing at a speed range of $0.1-2.5 \mathrm{~m} / \mathrm{s}$ in a pipe with a diameter range of 0.1-1 $\mathrm{m}$ (Water UK, 2003; Zabnieńska-Góra and Dudkiewicz, 2018).

The limitation in the range of $H_{0}$ is due to the flume dimensions. Higher initial heads $H_{0}$ were not possible due to the flume height, and lower initial heads did not produce sufficiently high water pressure to be read in the piezometric tubes.

The manufacturing procedure of the super-hydrophobic porous medium has been reported in Auwerter et al. (2019). The porous samples were formed by sintering of loose-packed particles of soda-Lime glass powder $\left(\mathrm{d}_{50}\right.$ of $18 \mu \mathrm{m}$ and $\mathrm{d}_{90}$ of $80 \mu \mathrm{m}$; Trovotech $\mathrm{GmbH}$, BitterfeldWolfen, Germany) in a crucible (dimensions $4.0 \times 9.6 \times 1.8 \mathrm{~cm}$ ) at $670^{\circ}$ for 30 minutes. The sintering of glass powder produced a porous surface with roughness heights up to $40 \mu \mathrm{m}$. Then, trichloro $(1 \mathrm{H}, 1 \mathrm{H}, 2 \mathrm{H}, 2 \mathrm{H}$ perfluorooctyl)silane was used as a surface modifying agent to change the surface chemistry and reduce the interface attraction between the water and the surface, resulting in a super-hydrophobic surface with a water contact angle of $153^{\circ}$ and 
TABLE 1: Conditions of experiments S1-3, C1-3 and T1-3.

$\operatorname{Exp} \quad H_{0}(\mathrm{~m}) \quad Q_{d}\left(\mathrm{~L} \mathrm{~min}^{-1}\right) \quad p_{a}(\mathrm{bar}) \quad \Delta t_{n b}(\min ) \quad \Delta t_{b}(\mathrm{~min}) \quad \operatorname{Re}(-) \times 10^{4}$

\begin{tabular}{llllccc}
\hline S1 & 0.22 & - & - & - & - & 4.02 \\
S2 & 0.24 & - & - & - & - & 4.45 \\
S3 & 0.27 & - & - & - & - & 4.75 \\
C1 & 0.22 & 1.5 & 0.10 & 0 & 300 & $4.38-4.46$ \\
C2 & 0.24 & 2.0 & 0.11 & 0 & 300 & $4.57-4.63$ \\
C3 & 0.27 & 2.2 & 0.15 & 0 & 300 & $4.77-4.81$ \\
T1 & 0.22 & 1.5 & 0.10 & 300 & 10 & $4.00-4.28$ \\
T2 & 0.24 & 2.0 & 0.11 & 300 & 10 & $4.40-4.46$ \\
T3 & 0.27 & 2.2 & 0.15 & 300 & 10 & $4.50-4.65$
\end{tabular}

$33.7 \%$ porosity.

The super-hydrophobic surface across the top surface of the duct was prepared from single tiles of dimensions $0.1 \mathrm{~m}$ (length) $\times 0.08 \mathrm{~m}$ (width) $\times 0.005 \mathrm{~m}\left(d_{s}\right.$, height). A total of 15 tiles were placed along the duct as the top wall. The tiles were glued with silicone sealant to a piece of acrylic of width $0.05 \mathrm{~m}$ placed on both right and hand sides along the duct as an extra support for the tiles. Thus, the width (w) of tiles exposed to the flow was $0.07 \mathrm{~m}$.

\section{RESULTS}

\section{Determination of $f_{d}$}

The head loss between two locations in an enclosure of constant cross-section $A$ and perimeter $P$ is given by (Nakayama, 2018):

$$
\Delta H=\bar{f}_{d} \frac{L}{D_{h}} \frac{U^{2}}{2 g}
$$

where $L$ is the distance between the two locations, $\bar{f}_{d}$ is the average (Darcy-Weissbach) friction factor of the duct and $D_{h}=4 A / P$ is the hydraulic diameter. The velocity can be inferred from the volume flow rate $Q$ as outlined in section 2 .

The head loss $\Delta H$ was measured in terms of the difference in the piezometric head $(\Delta h)$ between two piezometric tubes. Piezometric tubes at $0.343\left(x_{1}\right), 0.686 \mathrm{~m}\left(x_{2}\right)$ and $0.886 \mathrm{~m}$ $\left(x_{3}\right)$ from the upstream side of the duct were used to obtain the reading of the piezometric heads $h_{1}$ (at $\left.x_{1}\right), h_{2}$ (at $x_{2}$ ) and $h_{3}$ (at $x_{3}$ ), from which three $\Delta h$ were obtained from the difference $h_{1}-h_{2}, h_{1}-h_{3}$ and $h_{2}-h_{3}$. Thus, each replicate produced three data points. Each set of experiment was composed of triplicates, thus producing a total of nine data points for each experimental condition.

Experiments S1-3 (all surfaces made from acrylic) were performed first, and the results of $f_{d}$ of acrylic are presented in Table 2. Experiments S1-3 demonstrate that the mean value of $f_{d}$ is within $99.5 \%$ of the expected value $f_{B}$, thereby clearly confirming the accuracy of the 
TABLE 2: Friction coefficients $f_{d}$ for acrylic reference surface $\left(f_{0}\right)$, super-hydrophobic surface with bubble injection $\left(f_{b}\right)$, in Cassie-Baxter state $\left(f_{c}\right)$ and in Wenzel state $\left(f_{w}\right)$.

\begin{tabular}{|c|c|c|c|c|c|}
\hline & $\operatorname{Exp}$ & $\begin{array}{l}t \\
\text { (minutes) }\end{array}$ & $f_{d} \pm \operatorname{std}\left(\times 10^{-2}\right)$ & $f_{B}\left(\times 10^{-2}\right)$ & $\Delta f_{d}(\%)$ \\
\hline \multirow[t]{3}{*}{$f_{d 0}$} & $\mathrm{~S} 1$ & - & $2.19 \pm 0.18$ & 2.20 & $7.7^{1}$ \\
\hline & S2 & - & $2.19 \pm 0.10$ & 2.17 & $5.5^{1}$ \\
\hline & S3 & - & $2.13 \pm 0.14$ & 2.13 & $6.5^{1}$ \\
\hline \multirow[t]{3}{*}{$f_{b}$} & $\mathrm{C} 1$ & - & $1.40 \pm 0.08$ & 2.18 & -36 \\
\hline & $\mathrm{C} 2$ & - & $1.42 \pm 0.22$ & 2.16 & -34 \\
\hline & C3 & - & $1.58 \pm 0.08$ & 2.13 & -26 \\
\hline \multirow[t]{3}{*}{$f_{c}$} & $\mathrm{~T} 1$ & 0 & $1.53 \pm 0.06$ & 2.22 & -31 \\
\hline & $\mathrm{T} 2$ & 0 & $1.57 \pm 0.04$ & 2.22 & -29 \\
\hline & $\mathrm{T} 3$ & 0 & $1.52 \pm 0.15$ & 2.16 & -29 \\
\hline \multirow[t]{3}{*}{$f_{w}$} & $\mathrm{~T} 1$ & 300 & $2.74 \pm 0.06$ & 2.23 & +23 \\
\hline & $\mathrm{T} 2$ & 300 & $2.93 \pm 0.04$ & 2.20 & +33 \\
\hline & T3 & 300 & $2.81 \pm 0.15$ & 2.17 & +30 \\
\hline
\end{tabular}

${ }^{1}$ Denotes the accuracy of the measurement technique.

experimental setup, and indicate that the acrylic surface is smooth, and its friction factor can thus can thus be calculated using the Blasius equation for smooth surfaces at any $R e \geq$ 4,000 (Nakayama, 2018):

$$
f_{B}=\frac{0.316}{R e^{0.25}}
$$

In order to determine the friction factor for the top surface alone, it was assumed that the friction factor of all acrylic surfaces (sides and bottom) is given by (3), which leads to:

$$
f_{d}=\frac{\bar{f}_{d} P-f_{B}(P-w)}{w} .
$$

Calculated values of $f_{d}$ of the super-hydrophobic surface of experimental sets $\mathrm{C}$ and $\mathrm{T}$ are presented in Table 2. The results show a decrease in $f_{d}$ of $29-36 \%$, in accordance with previous studies (Park et al., 2015; Murai et al., 2007). The distribution of points is given at different $R e$ obtained by raising or lowering the initial head.

The reduction of $f_{d}$ using the continuous bubble injection technique was due to the air pockets created by the air bubbles. The air pockets lower the interfacial contact between the fluid and the surface of the duct, and the increased contact between the gas-liquid lowers $f_{d}$ due to the slip between the gas-liquid interface. Due to the continuous air bubble injection in experiments $\mathrm{C} 1-3, f_{d}$ is $26-36 \%$ lower than in experiments $\mathrm{S} 1-3$. 


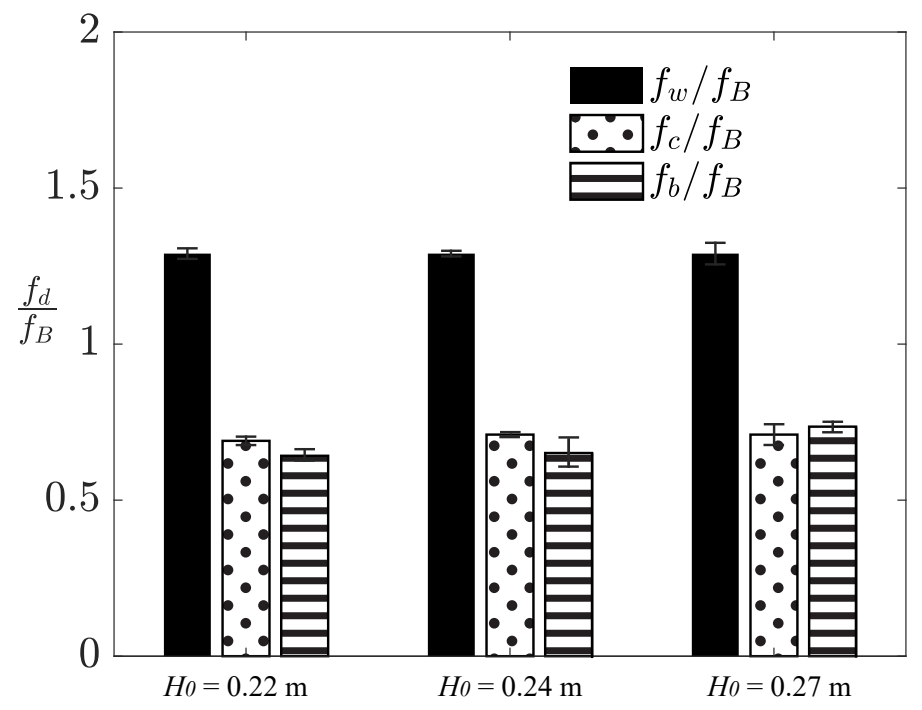

FIG. 4: Comparison of normalised friction factor of a smooth surface $\left(f_{B}\right)$ against the friction factor of the super-hydrophobic surface in the wetting state $f_{w}$, and the super-hydrophobic surface in the Cassie-Baxter state $f_{d}$ with and without continuous bubble injection.

Results of $f_{d}$ in the Cassie-Baxter state without continuous bubble injection are presented in Table 2 and show a reduction in $f_{d}$ of approximately 30\%. Measurements of $f_{d}$ were taken immediately after the air bubble injection was switched off. The super-hydrophobic surface holds the air pockets on the surface for a certain period without the continuous bubble injection. Without the slip effect of the air pockets the surface enters the Wenzel wetting state, and the friction factor increases to approximately $30 \%$ higher than $f_{B}$. Fig. 4 presents the normalised values of $f_{c}$ and $f_{b}$, indicating limited (10-15\%) difference between the reduction in the friction factor between the two sets, showing that with intermittent air injection it is possible to achieve similar levels of drag reductions without the energy demand required to inject air bubbles.

The friction coefficient in the Wenzel state $f_{w}$ can be used to estimate the relative roughness height $\epsilon$ of the surface by means of the Colebrook-White equation (Colebrook and White, 1937):

$$
\frac{1}{\sqrt{f_{d}}}=-2 \log \left\{\frac{\epsilon}{3.7 D_{h}}+\frac{2.51}{R e \sqrt{f_{d}}}\right\}
$$

Using the equation above for the values of $f_{w}$ reported in table 2 , indicates that $\epsilon$ is between $70 \pm 23 \mu \mathrm{m}$. In Auwerter et al. (2019) we obtained a mean value of $\epsilon=30 \mu \mathrm{m}$ by analysing the surface using X-ray micrographs obtained from a sample of dimensions $0.25 \times 0.5 \times 0.25$ $\mathrm{cm}$ because a high resolution of the surface structure was required, and consequently the sample size analysed was substantially smaller than the surface area applied in this study. Other studies of turbulent water flow on super-hydrophobic surfaces have applied engineered surface features of similar size range of 30-60 $\mu \mathrm{m}$ (Daniello et al., 2009). The size of features in super-hydrophobic surfaces of random surface structures presented in other studies range from $1 \mu \mathrm{m}$ to $1 \mathrm{~mm}$ (Abu Rowin et al., 2018; Crick and Parkin, 2009). 


\section{Durability of the superhydrophobic surface and recovery of the Cassie-Baxter state}

The evolution of the friction factor over time for all three experiments is presented in Fig. 5. Immediately after the superhydrophobic surface had been fully restored to the Cassie-Baxter state by blowing air through the surface, at $t=0$, the experiments have a friction coefficient equal to $f_{c}$. As time passes, the air pockets required for maintaining the Cassie-Baxter state disappear, thereby increasing $f_{d}$, passing the friction factor of an equivalent smooth surface $\left(f_{B}\right)$ and settling onto the friction coefficient associated with the Wenzel state $f_{w}$ due to the surfaces roughness. Once an air pressure was applied across the super-hydrophobic surface (see secondary axis in Fig. 5) at the specified times in Table 2, the Cassie-Baxter state was recovered, as evident from the drop in $f_{d}$ similar to that in the previous cycle. This is evidence of the capability of the material to recover the Cassie-Baxter state under turbulent conditions, confirming the results presented in Auwerter et al. (2019) under static pressure.

The reference smooth wall value $f_{B}$ (dashed line in Fig. 5) is a useful indicator for the durability of the friction-reduction. The friction coefficients $f_{d}$ are equal to $f_{B}$ at different times for the three experiments, indicating that the durability of the Cassie-Baxter state depends strongly on the water pressure applied to the surface. Values of $f_{d}$ show that once the top surface has transitioned from the Cassie-Baxter to the Wenzel state, $f_{d}$ remains constant, and the values of $f_{d}$ are then equivalent to that of a normal rough surface, as discussed in the previous section. More details of a best-fit of $f_{d}(t)$ with an analytical expression (solid line in Fig. 5) are presented in the next section.

In Exp. T1 $f_{d}$ increased slowly, and the Cassie-Baxter state was retained for 1 hour. The initial friction factor value $f_{c}$ was 0.0153 and increased gradually. At $t=40$ minutes $f_{d}$ became close to $f_{B}$. The trend-line suggests that $f_{d}$ becomes equal to $f_{B}$ at $t=1$ hour. At $\mathrm{t}=3$ hours the surface had transitioned entirely to the Wenzel state and $f_{d}$ increased to 0.0274 , showing an increase of $1.8 f_{c}$. After five hours, the air later was replenished by gently blowing air through the surface for 10 minutes. After the air flow was stopped, $f_{c}$ was 0.0179 , returning to values below the dashed $f_{B}$ line and recovering the Cassie-Baxter state, but not fully to the initial value of 0.0153 , i.e. an increase of $17 \% f_{c}$. After the first recovery of the Cassie-Baxter state, $f_{d}$ increase slowly once more and crossed $f_{B}$ line after 1 hour from $f_{c}=0.0179$. At $t=10.25 \mathrm{hr}$ an air pressure was applied once more and $f_{c}$ decreased to 0.0150, showing the full recovery of the Cassie-Baxter state for the second time.

In Exp. T2 the surface transitioned from the Cassie-Baxter to the Wenzel state much more rapidly. The initial $f_{c}$ value in Fig. $5 \mathrm{~b}$ was 0.0157 and crossed the $f_{B}$ line between $\mathrm{t}=$ 35 and 40 minutes, as suggested by the trend-line. At $t=3$ hours the Cassie-Baxter effect was completely depleted and $f_{d}$ increased to 0.0293 , showing an increase of $1.9 f_{c}$. After replenishing the air layer $t=5$ hours, $f_{d}$ decreased to 0.0170 , similarly to the case presented in Fig. 5a. After the first recovery of the Cassie-Baxter state, $f_{c}$ increased slowly once more and crossed $f_{B}$ line after 30 minutes from $f_{c}=0.0170$. The second cycle between $t=5$ to 10 $\mathrm{hr}$ deviates from the data points between $\mathrm{t}=0$ and 5 hours. The data points after $f_{d}$ crossed $f_{B}$ line do not increase by as much as $1.9 f_{c}$, but by $1.6 f_{c}$ at $\mathrm{t}=5$ hours. A second recovery of the Cassie-Baxter state occurred at $t=10.25$ hours after a second air replenishment event after which $f_{d}$ decreased to 0.0176 . This is higher than the first and second $f_{c}$ immediately 
a)

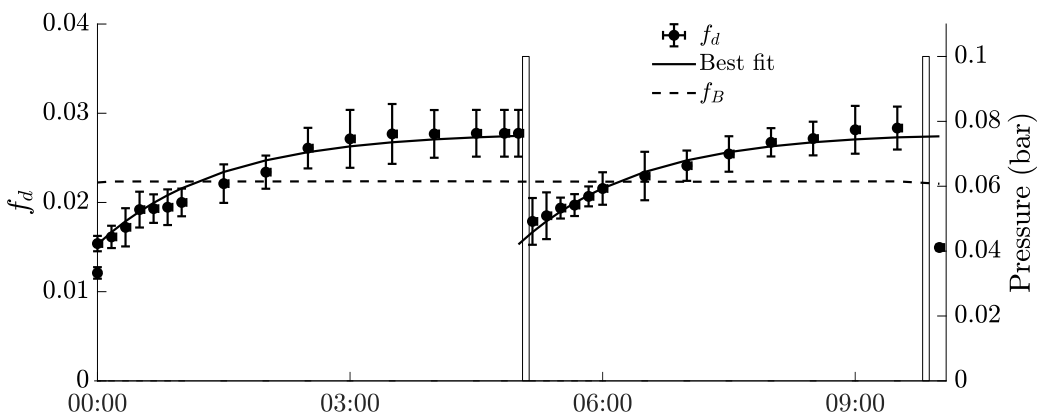

b)

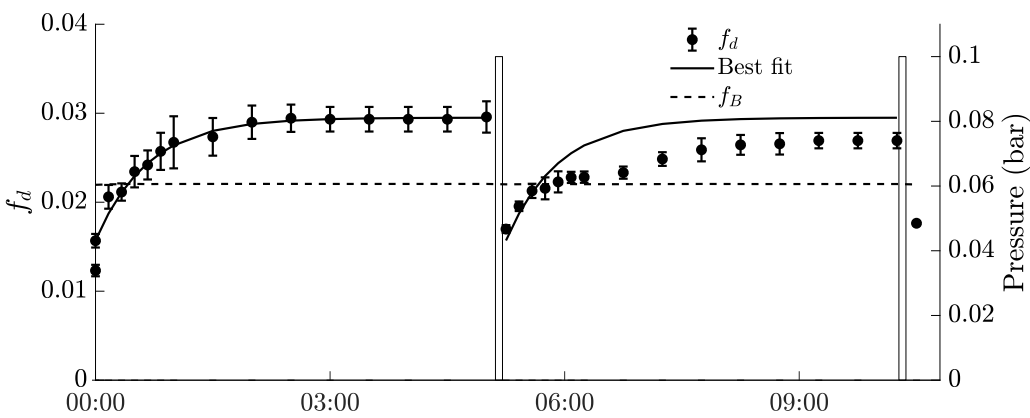

c)

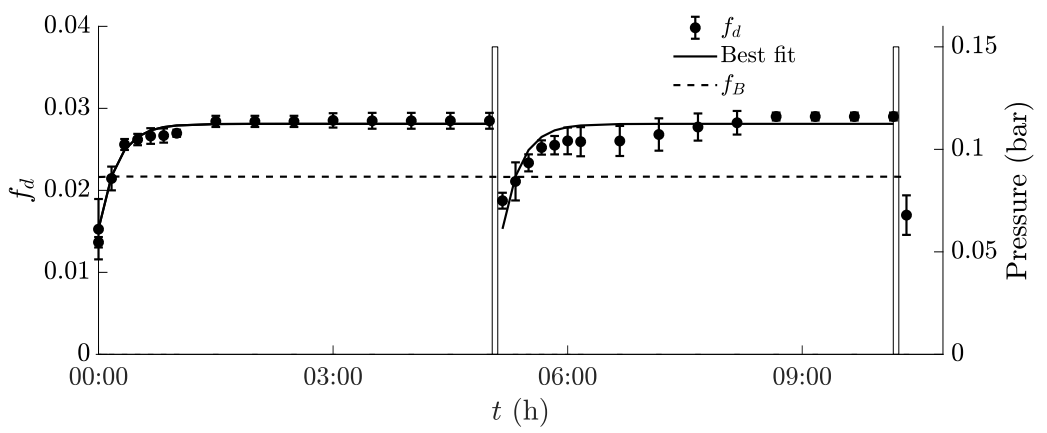

FIG. 5: Evolution of $f_{d}$ over time for experiment T1 (a), T2 (b) and T3 (c). The lowest data point at the initial time correspond to the values of $f_{d}$ in C1-3. The data points are the measured values for $f_{d}$, the dashed line is the smooth wall value calculated using eq. 3 . The solid line is the best fit of (8) to the first cycle for each case. The secondary $y$ axis denotes the pressure in the air chamber above the surface, and the bar plot shows when air pressure was applied at $t=5 \mathrm{~h}$ and $10 \mathrm{~h}$.

taken after the first air pressure was applied.

In Exp. T3 $f_{d}$ increased rapidly and crossed the $f_{B}$ line in under only 10 minutes. The initial friction factor $f_{c}$ was 0.0152 and increased to a maximum of $0.0281\left(1.8 f_{c}\right)$ at approximately $\mathrm{t}=1.5$ hours. At $t=5$ hours an air pressure of 0.15 bar was applied and $f_{d}$ decreased to $0.0187,23 \%$ higher than $f_{c}$. In this experiment, a higher pressure was applied in order to inject air through the porous surface because it was observed that with 0.1 bar there were no bubbles leaving the surface. After the recovery of the Cassie-Baxter state, $f_{c}$ increased rapidly again, crossing $f_{B}$ line in under 10 minutes and up to a maximum of 0.0290 at $\mathrm{t}=9$ hours (4 hours after $f_{d}$ decreased to 0.0187 ). At $\mathrm{t}=10.25$ an air pressure of 0.15 
bar was applied again and $f_{c}$ decreased to 0.0170 , which is $12 \%$ higher than $f_{c}$ in the first cycle, but $20 \%$ lower than $f_{B}$, indicating the recovery of the Cassie-Baxter state.

The second cycle at $t=5$-10 hours shows that for experiment $\mathrm{T} 1$, the material displays fully repeatable behaviour, demonstrating the recoverability of the super-hydrophobicity. For experiments T2 and T3, the deviations with the first cycle are larger, although the period $T$ over which the material transitions to the Wenzel state remains roughly the same. The difference in $f_{c}$ between the first and second cycles shows that the air pressure and time applied to the recovery of the Cassie-Baxter state was not homogeneous and sufficient to recover the super-hydrophobicity in all parts of the super-hydrophobic top layer. The full recoverability of the air pockets was, however, obtained between replicates as the material could dry overnight.

\section{Can durability be explained from the porous nature of the material?}

In this section we will develop a simple model which relates the durability of the superhydrophobicity to the porous properties of the material. The flow through a porous medium is given by the Darcy equation:

$$
q=-\frac{\kappa \Delta p}{\mu d_{S}}
$$

where $q$ is the Darcy velocity, $\kappa$ is the permeability coefficient, $\Delta p$ is the pressure difference $\left(p_{w}-p_{a}\right)$ across the porous medium, $d_{S}$ is its thickness and $\mu$ is the dynamic viscosity of the fluid.

In order to determine the reference permeability of the superhydrophobic surface, the measurements taken during the air replenishment phase can be used. In this case, the Darcy velocity $q$ can be obtained directly from the air flow rate $Q_{d}$. The pressure difference can be calculated from the the gauge pressure $p_{a}$ in the air and in the water $p_{w}$. The latter was calculated from piezometric head reading at $x=0.343 \mathrm{~m}$ and using $h=p_{w} / \rho_{w} g+z$, where $z$ is the elevation head, which was taken as $z=\delta$ (half-channel height) to obtain $p_{w}$ at the surface of the duct. The dynamic viscosity of air is $\mu=1.8 \times 10^{-5} \mathrm{Ns} / \mathrm{m}^{2}$ at $20^{\circ} \mathrm{C}$. The estimated permeability coefficient $\kappa$ was $(4.8 \pm 1) \times 10^{-15} \mathrm{~m}^{2}$, which is of the same order of magnitude as other super-hydrophobic porous materials (Patel et al., 2012).

Using (6), the time for water to fill roughness elements of size $\epsilon$ on a rough surface can be argued to scale as

$$
T \sim \frac{\epsilon}{q}=\frac{\epsilon \mu d_{S}}{\kappa \Delta p}
$$

Hence, $T$ can expected to be inversely proportional to the pressure difference across the porous surface. Here it should be noted that time-dependence of the pressure inside the duct due to the loss of super-hydrophobicity is assumed to be a higher-order effect that will not be considered here.Indeed, the change in pressure inside the duct from the beginning to the end of the experiment, in terms of piezometric height, was on average $2 \mathrm{~mm}$ up to a maximum, on average, of $5 \mathrm{~mm}$ between all cases. This implies that the maximum relative change is 5 percent which can be safely assumed to be a higher-order effect.

In order to test the hypothesis that $T \sim \Delta p^{-1}$, we extract from the measurements a relaxation time $T$ in which the surface transitions from a Cassie-Baxter to a Wenzel state 
by means of a best-fit to the data of $f_{d}$ by assuming a form

$$
f_{d}=f_{w}+\left(f_{c}-f_{w}\right) \exp \left(-\frac{t}{T}\right)
$$

This expression provides a robust means to define the relaxation (e-folding) time $T$. The best fit was determined using the MATLAB Curve Fitting App using values of $T$ from the first cycle ( $t=0$ to 5 hours). The relaxation time $T$ was 60,30 and 10 minutes for experiments T1, T2 and T3, respectively. The correlation coefficient $\left(R^{2}\right)$ for each case was at least $\mathrm{R}^{2}>0.96$ and the result is shown in Fig. 5. The same best-fit solution is shown in the second cycle. It shows that for experiment $\mathrm{T} 1$, the material displays fully repeatable behaviour, demonstrating the recoverability of the super-hydrophobicity. For experiments T2 and T3, the deviations with the first cycle are larger, although the period $T$ over which the material transitions to the Wenzel state remains roughly the same.

The time $(T)$ of the Cassie-Baxter to Wenzel transition is compared in Fig. 6 with the pressure of water to which the super-hydrophobic surface was subjected. Fig. 6 shows the experimental results of $T$ for T1-3 plotted against $1 / \Delta p\left(p_{a}=0\right.$ in T1-3 at $t$ between 0-5 hours, therefore $\Delta p=p_{w}$ ). The data was fitted into a line following the linear equation $T=$ $1.97 \times 10^{5} / \Delta p-1.91 \times 10^{2}$ obtained by linear regression and $\mathrm{R}^{2} \geq 0.997$, which indicates good data correlation. From the fact that this correlation does not cross the origin, it can be concluded that the durability behaviour observed here is not consistent with air loss as a consequence of flow through the porous material.

The regression line shows the relaxation time $T$ tending to zero (Cassie-Baxter state lost immediately) at a pressure $p_{w}$ of $1.0314 \times 10^{3} \mathrm{~Pa}$, or $h=0.1051 \mathrm{~m}$ in terms of pressure head at the surface, which is only $5 \mathrm{~mm}$ higher than the maximum $h$ obtained from the piezometric tube reading at $\mathrm{x}=0.343 \mathrm{~m}$. This implies that there is a maximum pressure difference beyond which superhydrophobicity cannot be sustained. It was unfortunately not possible to apply a higher $p_{w}$ by raising the initial head $H_{0}$ due to physical limitations of the flume height. It can be inferred from Fig. 6 that the durability of the wetting state is linked to the pressure of water applied against the super-hydrophobic surface.

\section{CONCLUSION}

The durability of friction reduction of a porous super-hydrophobic surface that can recover the Cassie-Baxter wetting state by blowing air through the surface under turbulent flow conditions was demonstrated. In the Cassie-Baxter state, the material under consideration showed reductions in the friction factor $f_{d}$ of $29-36 \%$ relative to a standard hydraulically smooth surface. In the Wenzel state, the friction factor was 23-33 \% larger than for a smooth surface due to the surface roughness, which was estimated to be $70 \mu \mathrm{m}$. The Cassie-Baxter state could be recovered by gently blowing air through the super-hydrophobic surface for a short period of time.

It was shown that lifetime of the Cassie-Baxter state $T$ depends strongly on the pressure of water applied against the super-hydrophobic surface. Observed values of $T$ ranged from 10 to 60 minutes for a short head difference of only $5 \mathrm{~cm}$. For a standard porous surface, the relaxation time $T$ can be expected to scale inversely proportional to the pressure difference $\Delta p$ as $T=a \Delta p^{-1}$, where $a$ is a constant. However, for the material under consideration this behaviour was not observed. A best fit of the data agreed best with $T=a\left(\Delta p^{-1}-\Delta p_{\max }^{-1}\right)$, 


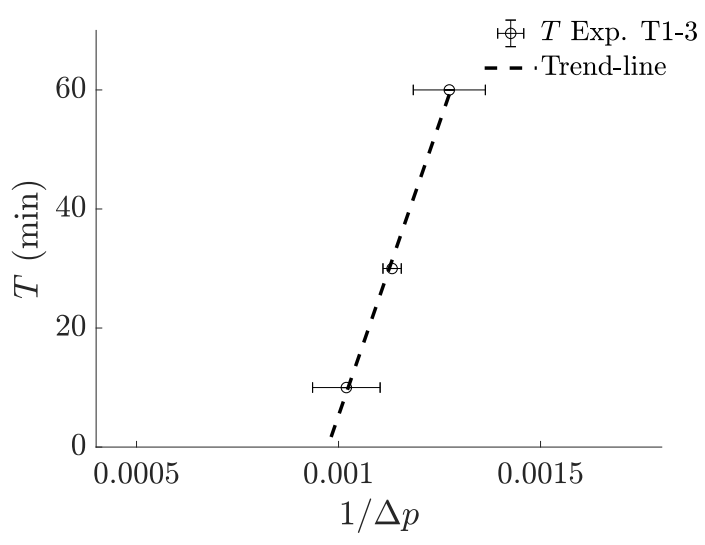

FIG. 6: Values of $T$ obtained at $\Delta p$ of Exp. T1-3. Horizontal error bars were obtained from variations in height in piezometric tubes. Vertical error bars are not visible because no variation in the relaxation time $T$ was observed. The dashed line is the linear regression following the linear equation $T=1.97 \times 10^{5} / \Delta p-1.91 \times 10^{2}$ and $\mathrm{R}^{2} \geq 0.997$.

where $a=1.97 \times 10^{5}$ and $\Delta p_{\max }=1.031 \times 10^{3} \mathrm{~Pa}$. This suggests that there is a critical pressure difference beyond which the Cassie-Baxter state cannot be sustained for the material under consideration. It was not possible to verify experimentally what happens at the critical pressure difference $\Delta p_{\max }$ due to the height of the flume in which the apparatus was placed which limits the initial head that can be applied. Future research should concentrate on understanding this behaviour, how it links with the surface properties and the flow regime (laminar/turbulent).

The current study has shown that the super-hydrophobic material under consideration (Auwerter et al., 2019) is able to recover the Cassie-Baxter state under fully immersed conditions. An important advantage of this material is that it is intrinsically hydrophobic it does not rely on a surface coating which can be removed. This makes the surface suitable to achieve drag reduction in situations where the material is immersed for extended periods of time. However, the application should be one where the pressure difference is relatively small, as the relaxation time $T$ was shown to be highly dependent on the pressure difference. This rules out, for the current time, applications in the water industry since the pressure heads inside water pipes far exceed those considered here.

It is recommended for future work to visualise the flow field on the transition $f_{c}$ to $f_{B}$ to obtain additional information about the durability of the air pockets and understand the effects of bubble size, distribution and depletion on the slip at the super-hydrophobic surface. Moreover, to improve the suitability of this system for practical applications, it is recommended to apply a control system that releases air bubbles based on feedback from drag measurements, such that low-drag performance can be guaranteed over extended time duration. Furthermore, it may be worth experimenting with the air pressure in the layer outside the pipe, which would allow for much larger base pressures inside the pipe.

\section{ACKNOWLEDGEMENTS}

We would like to acknowledge support from David De Ruyter for his direct technical help, and the UK Engineering and Physical Sciences Research Council (EPSRC) who fund 
Centre for Doctoral Training in Sustainable Civil Engineering in the Department of Civil and Environmental Engineering at Imperial College London via grant EP/L016826/1.

\section{DATA AVAILABILITY}

All data, models, or code generated or used during the study are available from the corresponding author by request. Data generated in this research and available upon request include: Measurements of piezometric head $h$ and volume flux $Q$ over time.

\section{NOMENCLATURE}

The following symbols are used in this paper:

$$
\begin{aligned}
& A=\text { Cross-sectional area }(\mathrm{m}) \\
& \mathrm{d}_{50}=\text { Median particle diameter } \\
& \mathrm{d}_{90}=90 \% \text { of the particles is smaller than this value } \\
& d_{s}=\text { Tile height }(\mathrm{m}) \\
& D=\text { Diameter }(\mathrm{m}) \\
& D_{h}=\text { Hydraulic diameter }(\mathrm{m}) \\
& \delta=\text { Half-channel height }(\mathrm{m}) \\
& \Delta h=\text { Piezometric head difference between two locations (m) } \\
& \Delta H=\text { Energy head loss }(\mathrm{m}) \\
& \Delta f_{d}=\text { Friction factor difference between two points } \\
& \Delta p=\text { Pressure difference }(\mathrm{Pa}) \\
& \Delta Q=\text { Flow difference between sets }\left(\mathrm{m}^{3} / \mathrm{s}\right) \\
& \Delta t_{b}=\text { Time with aeration (minutes) } \\
& \Delta t_{n b}=\text { Time without aeration (minutes) } \\
& f_{d}=\text { Friction factor } \\
& f_{0}=\text { Friction factor of a hydraulically smooth duct } \\
& f_{c}=\text { Friction factor in the Cassie-Baxter state } \\
& f_{w}=\text { Friction factor in the Wenzel state } \\
& f_{b}=\text { Friction factor in fully aerated conditions } \\
& \overline{f_{d}}=\text { Average friction factor } \\
& \epsilon=\text { Relative roughness height }(\mathrm{m}) \\
& h=\text { Piezometric head }(\mathrm{m}) \\
& H_{0}=\text { Initial head }(\mathrm{m}) \\
& \kappa=\text { Permeability coefficient }\left(\mathrm{m}^{2}\right) \\
& L=\text { Duct length }(\mathrm{m}) \\
& \mu=\text { Dynamic viscosity }\left(\mathrm{Ns} / \mathrm{m}^{2}\right) \\
& p_{a}=\text { Air pressure }(\mathrm{Pa}) \\
& p_{w}=\text { Pressure of water }(\mathrm{Pa}) \\
& P=\text { Perimeter }(\mathrm{m}) \\
& Q=\text { Volume flux }\left(\mathrm{m}^{3} / \mathrm{s}\right) \\
& Q_{d}=\text { Aeration volume flux }\left(\mathrm{m}^{3} / \mathrm{s}\right) \\
& q=\text { Darcy velocity }(\mathrm{m} / \mathrm{s}) \\
& R^{2}=\text { Correlation coefficient } \\
& R e=\text { Reynolds number } \\
& \rho=\text { Fluid density }\left(\mathrm{kg} / \mathrm{m}^{3}\right)
\end{aligned}
$$




$$
\begin{aligned}
t & =\text { Time }(\mathrm{s}) \\
T & =\text { Relaxation time }(\mathrm{s}) \\
U & =\text { Average flow velocity }(\mathrm{m} / \mathrm{s}) \\
w & =\text { Width }(\mathrm{m}) \\
x & =\text { Distance from beginning of duct }(\mathrm{m}) \\
z & =\text { Elevation head }(\mathrm{m}) .
\end{aligned}
$$

\section{REFERENCES}

Abu Rowin, W., Hou, J., and Ghaemi, S. (2018). "Turbulent channel flow over riblets with superhydrophobic coating." Experimental Thermal and Fluid Science, 94, 192-204.

Auwerter, L. C., Templeton, M. R., van Reeuwijk, M., Taiwo, O. O., and Cheeseman, C. (2019). "Development of porous glass surfaces with recoverable hydrophobicity." Materials Letters: X, 1, 100002.

Bennett, D. and Glaser, R. (2011). "Common pitfalls in hydraulic design of large diameter pipelines: Case studies and good design practice." Pipelines, 961-971.

Bobji, M. S., Kumar, S. V., Asthana, A., and Govardhan, R. N. (2009). "Underwater sustainability of the "cassie" state of wetting." Langmuir, 25(20), 12120-12126.

Boinovich, L. B., Emelyanenko, A. M., Emelyanenko, K. A., and Maslakov, K. I. (2016). "Anti-icing properties of a superhydrophobic surface in a salt environment: an unexpected increase in freezing delay times for weak brine droplets." Phys. Chem. Chem. Phys., 18, $3131-3136$.

Brassard, J.-D., Sarkar, D. K., and Perron, J. (2015). "Studies of drag on the nanocomposite superhydrophobic surfaces." Applied Surface Science, 324, 525-531.

Brennan, J. C., Geraldi, N. R., Morris, R. H., Fairhurst, D. J., McHale, G., and Newton, M. I. (2015). "Flexible conformable hydrophobized surfaces for turbulent flow drag reduction." Scientific Reports, 5, 10267.

Colebrook, C. F. and White, C. M. (1937). "Experiments with fluid friction in roughened pipes." Proceedings of the Royal Society of London. Series A, Mathematical and Physical Sciences, 161(906), 367-381.

Costantini, R., Mollicone, J.-P., and Battista, F. (2018). "Drag reduction induced by superhydrophobic surfaces in turbulent pipe flow." Physics of Fluids, 30(2), 025102.

Crick, C. R. and Parkin, I. P. (2009). "A single step route to superhydrophobic surfaces through aerosol assisted deposition of rough polymer surfaces: duplicating the lotus effect." Journal of Materials Chemistry, 19(8), 1074-1076.

Daniello, R. J., Waterhouse, N. E., and Rothstein, J. P. (2009). "Drag reduction in turbulent flows over superhydrophobic surfaces." Physics of Fluids, 21(8), 085103. 
Davis, A. M. J. and Lauga, E. (2009). "Geometric transition in friction for flow over a bubble mattress." Physics of Fluids, 21(1), 011701.

Du, P., Wen, J., Zhang, Z., Song, D., Ouahsine, A., and Hu, H. (2017). "Maintenance of air layer and drag reduction on superhydrophobic surface." Ocean Engineering, 130, 328 $-335$.

Fukuda, K., Tokunaga, J., Nobunaga, T., Nakatani, T., Iwasaki, T., and Kunitake, Y. (2000). "Frictional drag reduction with air lubricant over a super-water-repellent surface." Journal of Marine Science and Technology, 5(3), 123-130.

Gogte, S., Vorobieff, P., Truesdell, R., Mammoli, A., van Swol, F., Shah, P., and Brinker, C. J. (2005). "Effective slip on textured superhydrophobic surfaces." Physics of Fluids, $17(5), 051701$.

Guan, N., Liu, Z., Jiang, G., Zhang, C., and Ding, N. (2015). "Experimental and theoretical investigations on the flow resistance reduction and slip flow in super-hydrophobic micro tubes." Experimental Thermal and Fluid Science, 69, 45-57.

IEA (2016). Water Energy Nexus. International Energy Agency, <https://www.iea.org/publications/freepublications/publication/WorldEnergyOutlook2016ExcerptWat

Min, T. and Kim, J. (2004). "Effects of hydrophobic surface on skin-friction drag." Physics of Fluids, 16(7), L55-L58.

Moaven, K., Rad, M., and Taeibi-Rahni, M. (2013). "Experimental investigation of viscous drag reduction of superhydrophobic nano-coating in laminar and turbulent flows." Experimental Thermal and Fluid Science, 51, 239-243.

Mohammadi, R., Wassink, J., and Amirfazli, A. (2004). "Effect of surfactants on wetting of super-hydrophobic surfaces." Langmuir, 20(22), 9657-9662.

Moody, L. (1944). "Friction factors for pipe flow." Trans. ASME, 66(8), 671-677.

Murai, Y., Fukuda, H., Oishi, Y., Kodama, Y., and Yamamoto, F. (2007). "Skin friction reduction by large air bubbles in a horizontal channel flow." International Journal of Multiphase Flow, 33(2), 147-163.

Nakayama, Y. (2018). 7. Flow in Pipes. Elsevier, $<$ https://app.knovel.com/hotlink/khtml/id:kt011PH9L1/introduction-fluidmechanics/flow-in-pipes $>$.

Navier, C. (1823). "Memoire sur les lois du mouvement des fluids." l'Académie Royale des Sciences de l'Institut de France, Mém. Acad. R. Sci. Inst. Fr., 6, 389-440.

Nosonovsky, M. and Bhushan, B. (2008). Friction, Superhydrophobicity, and Biomimetics. Springer-Verlag Berlin Heidelberg. 
Paik, B.-G., Pyun, Y.-S., Kim, K.-Y., Jung, C.-M., and Kim, C.-G. (2015). "Study on the micro-dimpled surface in terms of drag performance." Experimental Thermal and Fluid Science, 68, 247-256.

Park, H. J., Tasaka, Y., Oishi, Y., and Murai, Y. (2015). "Drag reduction promoted by repetitive bubble injection in turbulent channel flows." International Journal of Multiphase Flow, 75, 12-25.

Patel, S. U., Manzo, G. M., Patel, S. U., Kulkarni, P. S., and Chase, G. G. (2012). "Permeability of electrospun superhydrophobic nanofiber mats." Journal of Nanotechnology, 2012,7 .

Shirtcliffe, N. J., McHale, G., Newton, M. I., and Zhang, Y. (2009). "Superhydrophobic copper tubes with possible flow enhancement and drag reduction." ACS Applied Materials and Interfaces, 1(6), 1316-1323.

Walker, G. M., Albadarin, A. B., McGlue, A., Brennan, S., and Bell, S. E. J. (2016). "Analysis of friction factor reduction in turbulent water flow using a superhydrophobic coating." Progress in Organic Coatings, 90, 472-476.

Wang, B., Wang, J., Dou, Z., and Chen, D. (2014). "Investigation of retention of gases in transverse hydrophobic microgrooved surfaces for drag reduction." Ocean Engineering, 79, $58-66$.

Water UK (2003). "The choice of pressure rating for polyethylene pipe systems for water supply and sewage duties." Water Industry Information and Guidance Note.

Xu, M., Sun, G., and Kim, C.-J. (2014). "Infinite lifetime of underwater superhydrophobic states." Phys. Rev. Lett., 113, 136103.

Yao, Y., Luo, J.-l., Liu, H., and Zhu, K. (2015). "Water tunnel experimental investigation on drag reduction of coating surface wall." Procedia Engineering, 126, 247-253.

Ybert, C., Barentin, C., Cottin-Bizonne, C., Joseph, P., and Bocquet, L. (2007). "Achieving large slip with superhydrophobic surfaces: Scaling laws for generic geometries." Physics of Fluids, 19(12).

Zabnieńska-Góra, A. and Dudkiewicz, E. (2018). "Analysis of water flow velocity criteria for the selection of copper pipes on the example of production buildings." E3S Web Conf., 46, 00007.

Zhang, J., Yang, S., and Liu, J. (2018). "Numerical investigation of a novel device for bubble generation to reduce ship drag." International Journal of Naval Architecture and Ocean Engineering, 10(5), $629-643$. 